\title{
Comparison of Quantities of Information in the Human Memory
}

\author{
Angel Angelov Dishliev a, Christian Assenov Girginov ${ }^{b}$, Katya Georgieva Dishlieva ${ }^{c}$ \\ a, b University of Chemical Technology and Metallurgy-Sofia, Bulgaria, 8, bul. Kliment Ohridski \\ ${ }^{c}$ Technical University of Sofia, Bulgaria, 8, bul. Kliment Ohridski \\ aadishliev@uctm.edu, girginov@uctm.edu, kgd@tu-sofia.bg
}

\begin{abstract}
A mathematical model of changing the amount of information in the abstract human memory is proposed in the presence of the subsequent "external discrete" training (filling the information). Under this model, the amount of information is a solution of impulsive differential equation with fixed moments of impulsive effects and variable structure. Sufficient conditions are proposed related to the moments and magnitudes of the impulsive effects (i.e., to the moments of discrete training and the volume of the received information), where the quantities of information in two different models of learning can be compared.
\end{abstract}

Indexing terms/Keywords: Information, Impulsive differential equation.

\section{Mathematics Subject Classification: 34A37}

\section{Supporting Agencies:}

1. Bulgarian National Scientific Research Fund, contract DM19/1 (2017).

2. Project BG05M2OP001-2.009-0015, University of Chemical Technology and Metallurgy-Sofia, Bulgaria.

Language: English

Date of Publication: 2018-27-10

ISSN: 2347-1921

Volume: 14 Issue: 02

Journal: JOURNAL OF ADVANCES IN MATHEMATICS

Website: https://cirworld.com

c) (7)

This work is licensed under a Creative Commons Attribution 4.0 International License.

How to Cite: Dishliev, A., Girginov, C., \& Dishlieva, K. (2018). Comparison of Quantities of Information in the Human Memory. JOURNAL OF ADVANCES IN MATHEMATICS, 14(2), 8004-8012. Retrieved from https://cirworld.com/index.php/jam/article/view/7840 


\section{Introduction}

In the articles [7], [8], and [10] the following concepts are introduced:

- Quantity of information $I=I(t)$, calculated at the moment $t>t_{0}$, where $t_{0} \geq 0$ is an initial moment;

- Information storage coefficient $\alpha=\alpha(t), t \geq 0$;

- Discreet, external information (in the form of short-term training)

$$
\Delta I(t)=I(t+0)-I(t-0)=I(t+0)-I(t), \quad t=t_{1}, t_{2}, \ldots,
$$

where the moments $t_{1}, t_{2}, \ldots$ are fixed in advance and $t_{0}<t_{1}<t_{2}<\ldots$;

- Coefficient of proportional completing the information at each training $\beta=\beta(t, I(t))>0, t=t_{1}, t_{2}, \ldots$.

In the above cited articles, the following hypotheses are used:

First hypothesis: The output numerical data and the obtained results are averaged, i.e. they refer to a "typical generalized representative" chosen by a group of learners placed under the same external conditions.

Second hypothesis: Information is quantified and its values at $t \geq t_{0}$ are expressed by the function $I=I(t)>0$.

Third hypothesis: Every external filling of the information in the human memory (which takes place through an organized training) is realized in a relatively short time period. Therefore, we will assume that the changing of information by the external effects is done instantaneously in the form of impulses at the fixed moments $t_{1}, t_{2}, \ldots, t_{0}<t_{1}<t_{2}<\ldots$. This means that the amount of information $I(t)$ in the case of short-terms discrete training is a peace-wise continuous function in $t \geq t_{0}$ with the points of discontinuity $t_{1}, t_{2}, \ldots$.

Here, the new hypotheses are introduced:

Fourth hypothesis: The internal (continuous) change in the amount of information in each interval $t_{i-1}<t \leq t_{i}, \quad i=1,2, \ldots$, is proportional to the volume of information. The coefficients of proportionality in these intervals are expressed by the functions $\alpha_{i-1}=\alpha_{i-1}(t), i=1,2, \ldots$, defined in $t \geq 0$.

Fifth hypothesis: After each training, the increase of the amount of information satisfies the equalities

$$
\Delta I\left(t_{i}\right)=I\left(t_{i}+0\right)-I\left(t_{i}\right)=I_{i}, \quad i=1,2, \ldots,
$$

where the positive constants $I_{1}, I_{2}, \ldots$ are given in advance.

Sixth hypothesis: The amount of information in the human memory is limited above, i.e. there is a memory capacity. The increase in the amount of information as a result of short-term discrete training is limited from below, i.e. we have a minimal intake of information.

\section{PRELIMINARY REMARKS}

In the article [10], formulated above Fifth hypothesis is replaced by the supposition:

Hypothesis [10]: After each training, the quantity increase of information satisfies the equality

$$
\Delta\left(t_{i}\right)=\beta\left(t_{i}, I\left(t_{i}-0\right)\right) \cdot I\left(t_{i}-0\right)=\beta\left(t_{i}, I\left(t_{i}\right)\right) . I\left(t_{i}\right), \quad i=1,2, \ldots,
$$


where $\beta=\beta(t, I(t))>0, t \geq 0$, is a coefficient (function determined in advance) of the proportional filling of the information.

Clear that, the Fifth hypothesis formulated here is a special case of Hypothesis [10]. For this purpose, it is enough to substitute

$$
\beta\left(t_{i}, I\left(t_{i}\right)\right)=I_{i} / I\left(t_{i}\right), i=1,2, \ldots,
$$

in equality (2) in order to obtain equality (1) of the Fifth hypothesis.

In the papers [8] and [10], we assume that the coefficients of proportionality in each intervals $t_{i-1}<t \leq t_{i}, \quad i=1,2, \ldots$, is the same, i.e. the equalities $\alpha_{0}(t)=\alpha_{1}(t)=\ldots, t \geq 0$ are valid.

In [10] the general problem that modeled the dynamics of the amount of information in the human memory at the presence of the relevant hypotheses is found. We have:

$$
\begin{aligned}
& \frac{d I}{d t}=\alpha\left(t-t_{i}\right) I, \quad t_{i-1}<t \leq t_{i} ; \\
& \Delta I\left(t_{i}\right)=\beta\left(t_{i}, I\left(t_{i}\right)\right) \cdot I\left(t_{i}\right), i=1,2, \ldots ; \\
& I\left(t_{0}\right)=I_{0} .
\end{aligned}
$$

In this study, provided that the five hypotheses formulated in the preceding paragraph are valid, as a special case of the mathematical model in [10], we can write the following initial value problem of differential equations with impulses that model the dynamics of the amount of information in the human memory:

$\frac{d I}{d t}=\alpha_{i-1}\left(t-t_{i}\right) I, \quad t_{i-1}<t \leq t_{i}$

$\Delta I\left(t_{i}\right)=I_{i}, \quad i=1,2, \ldots$

$I\left(t_{0}\right)=I_{0}$

The problem above is a main object of this paper. We denote its solution by $I\left(t ; t_{0}, t_{1}, \ldots, I_{0}, I_{1}, \ldots\right)$. The next inequalities are valid:

$I_{0}=I\left(t_{0} ; t_{0}, t_{1}, \ldots, I_{0}, I_{1}, \ldots\right) ; \quad I\left(t ; t_{0}, t_{1}, \ldots, I_{0}, I_{1}, \ldots\right) \geq 0, t \geq t_{0}$.

We will use the notation $I\left(t ; t_{0}, I_{0}\right)=I\left(t ; t_{0}, t_{1}, \ldots, I_{0}, I_{1}, \ldots\right)$ if the solution is not subjected to the impulsive effect up to the moment $t>t_{0}$. If the impulsive effects are in number $i$ to the same moment $t>t_{0}$, we will use also the notation $I\left(t ; t_{0}, t_{1}, \ldots, t_{i}, I_{0}, I_{1}, \ldots, I_{i}\right)=I\left(t ; t_{0}, t_{1}, \ldots, I_{0}, I_{1}, \ldots\right)$ for the solution of problem (3), (4), (5). The general solution of the above initial value problem has the form (see [10]) 


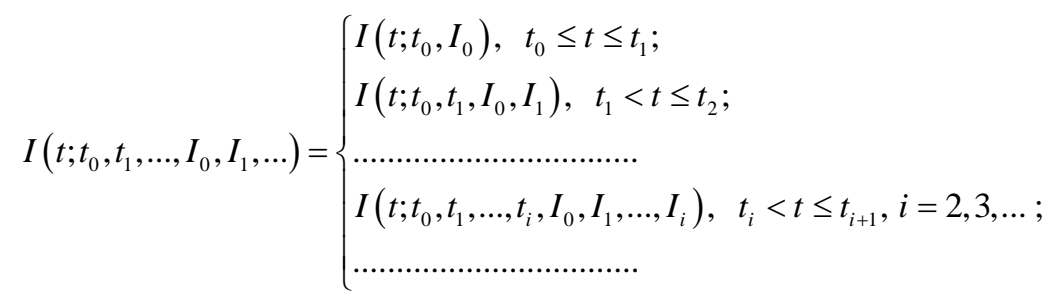

More precisely, referring to the right-hand side in (3)

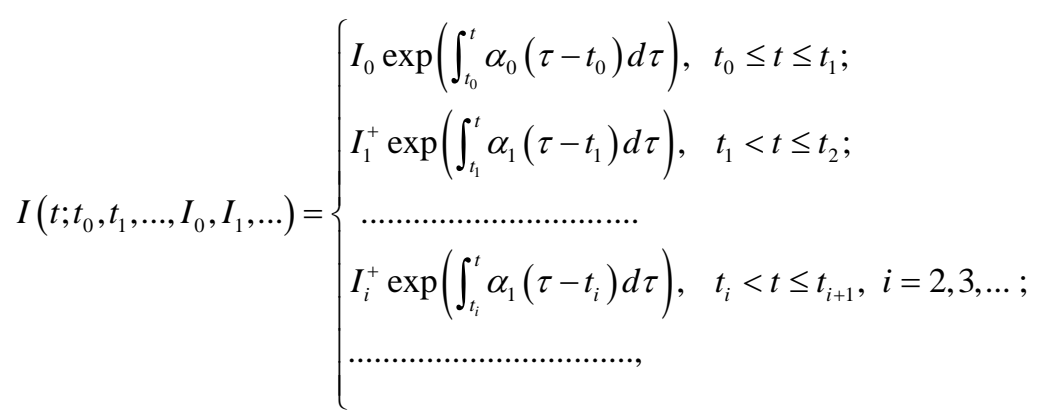

where:

$I_{i}^{+}=I\left(t_{i} ; t_{0}, t_{1}, \ldots, t_{i-1}, I_{0}, I_{1}, \ldots, I_{i-1}\right)+I_{i}, \quad i=1,2, \ldots$

The problem (3), (4), (5) is a typical for differential equations with impulsive effects and variable structure. The applications of this type equations are numerous (see [1] - [6], [9], [11] - [18]).

\section{MAIN RESULTS}

We will use the following conditions concerning the coefficients of storing information in the next two theorems:

$\mathrm{H} 1$. The functions $\alpha_{i-1}, i=1,2, \ldots$, are continuous with negative values for $t \geq 0$.

H2. It is fulfilled $\lim _{t \rightarrow \infty} \alpha_{i-1}(t)=0, \quad i=1,2, \ldots$.

In the next theorem, we will assume that the number of the impulsive impacts is a finite. Let the number of effects be $i$. Under this condition, we will consider two solutions of the problem (3), (4), (5), which differ not only in the impulsive moments but also in the magnitudes of the impulsive effects. The aim is to compare asymptotically the amount of information presented with these solutions.

\section{Theorem 1. Let:}

1. The Conditions $\mathrm{H} 1$ and $\mathrm{H} 2$ are satisfied.

2. The impulsive effects of Problem (3), (4) and (5) are finite.

Then:

- for each finite number impulsive effects $i$;

- for every two finite sequences of impulsive moments $0<t_{0}^{*}<t_{1}^{*}<\ldots<t_{i}^{*}$ and $0<t_{0}^{* * *}<t_{1}^{* * *}<\ldots<t_{i}^{* * *}$;

- for every two finite sequences of impulsive effects $I_{0}^{*}>0, I_{1}^{*}>0, \ldots, I_{i}^{*}>0$ and $I_{0}^{* *}>0, I_{1}^{* *}>0, \ldots, I_{i}^{* *}>0$,

for which it is fulfilled 


$$
I_{i}^{*+}=I\left(t_{i}^{*} ; t_{0}^{*}, t_{1}^{*}, \ldots, t_{i-1}^{*}, I_{0}^{*}, I_{1}^{*}, \ldots, I_{i-1}^{*}\right)+I_{i}^{*}<I\left(t_{i}^{* * *} ; t_{0}^{* * *}, t_{1}^{* *}, \ldots, t_{i-1}^{* *}, I_{0}^{* *}, I_{1}^{* *}, \ldots, I_{i-1}^{* *}\right)+I_{i}^{* *}=I_{i}^{* *+}
$$

there exists a constant $T, T>\max \left\{t_{i}^{*}, t_{i}^{* *}\right\}$, such that for each $t>T$, it is satisfied

$$
I\left(t ; t_{0}^{*}, t_{1}^{*}, \ldots, t_{i}^{*}, I_{0}^{*}, I_{1}^{*}, \ldots, I_{i}^{*}\right)<I\left(t ; t_{0}^{* *}, t_{1}^{* *}, \ldots, t_{i}^{* *}, I_{0}^{* *}, I_{1}^{* *}, \ldots, I_{i}^{* *}\right)
$$

Proof. Using equality (6) and inequality (7) for $t>\max \left\{t_{i}^{*}, t_{i}^{* *}\right\}$, we consistently obtain

$$
\begin{aligned}
I\left(t ; t_{0}^{*}, t_{1}^{*}, \ldots, t_{i}^{*}, I_{0}^{*}, I_{1}^{*}, \ldots, I_{i}^{*}\right) & -I\left(t ; t_{0}^{* *}, t_{1}^{* *}, \ldots, t_{i}^{* * *}, I_{0}^{* * *}, I_{1}^{* *}, \ldots, I_{i}^{* * *}\right) \\
& =I_{i}^{*+} \exp \left(\int_{t_{i}^{*}}^{t} \alpha_{i}\left(\tau-t_{i}^{*}\right) d \tau\right)-I_{i}^{* *+} \exp \left(\int_{t_{i}^{*}}^{t} \alpha_{1}\left(\tau-t_{i}^{* * *}\right) d \tau\right) \\
& =I_{i}^{*+} \exp \left(\int_{0}^{t-t_{i}^{*}} \alpha_{i}(\tau) d \tau\right)-I_{i}^{* *+} \exp \left(\int_{0}^{t-t_{i}^{* *}} \alpha_{i}(\tau) d \tau\right) \\
& =I_{i}^{*+} \exp \left(\int_{0}^{t-t_{i}^{* *}} \alpha_{i}(\tau) d \tau\right) \exp \left(\int_{t-t_{i}^{*}}^{t-t_{i}^{*}} \alpha_{i}(\tau) d \tau\right)-I_{i}^{* *+} \exp \left(\int_{0}^{t-t_{i}^{* *}} \alpha_{i}(\tau) d \tau\right) \\
& =\exp \left(\int_{0}^{t-t_{i}^{* *}} \alpha_{i}(\tau) d \tau\right)\left(I_{i}^{*+} \exp \left(\int_{t-t_{i}^{*}}^{t-t_{i}^{*}} \alpha_{i}(\tau) d \tau\right)-I_{i}^{* *+}\right)
\end{aligned}
$$

By the Conditions $\mathrm{H} 1$ and $\mathrm{H} 2$, we deduce that

$$
\begin{aligned}
& \lim _{t \rightarrow \infty} \int_{t-t_{i}^{*}}^{t-t_{i}^{*}} \alpha_{i}(\tau) d \tau=\left(t_{i}^{* *}-t_{i}^{*}\right) \lim _{t \rightarrow \infty} \alpha_{i}(\theta)=\left(t_{i}^{* * *}-t_{i}^{*}\right) \lim _{\theta \rightarrow \infty} \alpha_{i}(\theta)=0 \\
& \Rightarrow \lim _{t \rightarrow \infty} \exp \left(\int_{t-t_{i}^{*}}^{t-t_{i}^{*}} \alpha_{i}(\tau) d \tau\right)=1
\end{aligned}
$$

where the constant $\theta$ is between $t-t_{i}^{*}$ and $t-t_{i}^{* * * *}$. Then by (8), having in mind the equality (9), condition $\mathrm{H} 2$ and inequality (7), we find successively

$$
\begin{gathered}
\lim _{t \rightarrow \infty}\left(I\left(t ; t_{0}^{*}, t_{1}^{*}, \ldots, t_{i}^{*}, I_{0}^{*}, I_{1}^{*}, \ldots, I_{i}^{*}\right)-I\left(t ; t_{0}^{* *}, t_{1}^{* *}, \ldots, t_{i}^{* *}, I_{0}^{* *}, I_{1}^{* *}, \ldots, I_{i}^{* *}\right)\right) \\
\quad=\lim _{t \rightarrow \infty} \exp \left(\int_{0}^{t-t_{i}^{* *}} \alpha_{i}(\tau) d \tau\right)\left(I_{i}^{*+} \lim _{t \rightarrow \infty} \exp \left(\int_{t-t_{i}^{*}}^{t-t_{i}^{*}} \alpha_{1}(\tau) d \tau\right)-I_{i}^{* *+}\right) \\
\quad=\lim _{t \rightarrow \infty} \exp \left(\int_{0}^{t-t_{i}^{*}} \alpha_{i}(\tau) d \tau\right)\left(I_{i}^{*+}-I_{i}^{* *+}\right)<0 .
\end{gathered}
$$

Since the solutions $I\left(t ; t_{0}^{*}, t_{1}^{*}, \ldots, t_{i}^{*}, I_{0}^{*}, I_{1}^{*}, \ldots, I_{i}^{*}\right)$ and $I\left(t ; t_{0}^{* *}, t_{1}^{* *}, \ldots, t_{i}^{* *}, I_{0}^{* *}, I_{1}^{* *}, \ldots, I_{i}^{* *}\right)$ are continuous for $t>\max \left\{t_{i}^{*}, t_{i}^{* * *}\right\}$, then by the sign of the upper limit it follows that there is a constant $T>\max \left\{t_{i}^{*}, t_{i}^{* *}\right\}$, such that for each $t \geq T$, the next inequality is valid

$$
I\left(t ; t_{0}^{*}, t_{1}^{*}, \ldots, t_{i}^{*}, I_{0}^{*}, I_{1}^{*}, \ldots, I_{i}^{*}\right)<I\left(t ; t_{0}^{* * *}, t_{1}^{* * *}, \ldots, t_{i}^{* *}, I_{0}^{* *}, I_{1}^{* *}, \ldots, I_{i}^{* *}\right) .
$$

The theorem is proved.

The following statement is an important consequence of the above theorem. 
Tеорема 2. Assume that:

1. The conditions $\mathrm{H} 1$ and $\mathrm{H} 2$ are satisfied.

2. The impulsive effects of Problem (3), (4) and (5) are finite.

Then:

- for each finite number impulsive effects $i$;

- for each finite sequence of impulsive moments $0<t_{0}<t_{1}<\ldots<t_{i-1}$;

- for every two impulsive moments $t_{1}^{*}$ and $t_{i}^{* *}, t_{i-1}<t_{i}^{*}<t_{i}^{* *}$;

- for every finite sequence of impulsive effects $I_{0}>0, I_{1}>0, \ldots, I_{i}>0$,

there exists a constant $T, T>t_{i}^{* *}$, such that for each $t \geq T$, it is fulfilled

$$
I\left(t ; t_{0}, t_{1}, \ldots t_{i-1}, t_{i}^{*}, I_{0}, I_{1}, \ldots, I_{i}\right)>I\left(t ; t_{0}, t_{1}, \ldots t_{i-1}, t_{i}^{* * *}, I_{0}, I_{1}, \ldots, I_{i}\right)
$$

Proof. As $t_{i}^{*}<t_{i}^{* * *}$ and $\alpha_{i}(t)<0$ at $t \geq 0$, it follows that

$$
\begin{aligned}
I\left(t_{i}^{*} ; t_{0}, t_{1}, \ldots t_{i-1}, I_{0}, I_{1}, \ldots, I_{i-1}\right) \\
\quad=I_{i-1}^{+} \exp \left(\int_{t_{i-1}}^{t_{i}^{*}} \alpha_{i-1}\left(\tau-t_{i-1}\right) d \tau\right) \\
>I_{i-1}^{+} \exp \left(\int_{t_{i-1}}^{t_{i}^{*}} \alpha_{i-1}\left(\tau-t_{i-1}\right) d \tau\right) \\
\quad=I\left(t_{i}^{* * *} ; t_{0}, t_{1}, \ldots t_{i-1}, I_{0}, I_{1}, \ldots, I_{i-1}\right) \\
\Leftrightarrow I_{i}^{*+}=I\left(t_{i}^{*} ; t_{0}, t_{1}, \ldots t_{i-1}, I_{0}, I_{1}, \ldots, I_{i-1}\right)+I_{i} \\
>I\left(t_{i}^{* * *} ; t_{0}, t_{1}, \ldots t_{i-1}, I_{0}, I_{1}, \ldots, I_{i-1}\right)+I_{i}=I_{i}^{* *+} .
\end{aligned}
$$

From the last inequality and Theorem 1, we reach the statement of Theorem 2.

We introduce the condition:

H3. It is satisfied $\int_{0}^{\infty} \alpha_{i-1}(\tau) d \tau=A_{i-1}=$ const $<0, \quad i=1,2, \ldots$.

Remark 1. The existence of limits $\lim _{t \rightarrow \infty} \alpha_{i-1}(t)=0, i=1,2, \ldots$, follow by the equalities

$$
\int_{0}^{\infty} \alpha_{i-1}(\tau) d \tau=A_{i-1}
$$

i.e. condition $\mathrm{H} 2$ follows by condition $\mathrm{H} 3$. This means that the statements of the above two theorems will not change if in their formulation, condition $\mathrm{H} 2$ is replaced by condition $\mathrm{H} 3$.

If the number of the short-term trainings is $i$, then the magnitude $I_{\infty}=I_{i}^{+} \exp \left(A_{i}\right)$ is called an amount of residual information in memory. If we experimentally determine the amount of residual information $I_{\infty}$, then a constant $A_{i}$ is defined immediately. For more details, see the article [7].

Given the Sixth hypothesis, we introduce the condition: 
H4. There exist positive constants $I_{\min }$ and $I_{\max }$ such that the following inequalities are valid $I_{1} \geq \mathrm{I}_{\min }, I_{2} \geq \mathrm{I}_{\min }, \ldots$ and $I_{1}^{+} \leq I_{\max }, I_{2}^{+} \leq I_{\max }, \ldots$.

H5. The inequalities $A_{i} \geq A_{i-1}+\ln \frac{I_{\max }}{I_{\min }}, i=1,2, \ldots$ are fulfilled.

Theorem 3. Let:

1. The Conditions $\mathrm{H} 1, \mathrm{H} 3, \mathrm{H} 4$ and $\mathrm{H} 5$ are satisfied.

2. The impulsive effects of initial value problem (3), (4), (5) are finite.

Consider two solutions to the problem (3), (4), (5):

- $\quad I\left(t ; t_{0}, t_{1}, \ldots t_{i-1}, I_{0}, I_{1}, \ldots, I_{i-1}\right)$ with $i-1$ number of impulsive effects;

- $\quad I\left(t ; t_{0}, t_{1}, \ldots, t_{i}, I_{0}, I_{1}, \ldots, I_{i}\right)$ with $i$ number of impulsive effects.

Then:

- for each $i$;

- for every finite sequence of impulsive moments $0<t_{0}<t_{1}<\ldots<t_{i}$;

- for every finite sequence of impulsive effects $I_{0}>0, I_{1}>0, \ldots, I_{i}>0$,

there exists a constant $T, T>t_{i}$, such that for every $t \geq T$, it is fulfilled

$$
I\left(t ; t_{0}, t_{1}, \ldots, t_{i}, I_{0}, I_{1}, \ldots, I_{i}\right)>I\left(t ; t_{0}, t_{1}, \ldots t_{i-1}, I_{0}, I_{1}, \ldots, I_{i-1}\right)
$$

Proof. For $t>t_{i}$, we have

$$
\begin{aligned}
& I\left(t ; t_{0}, t_{1}, \ldots, t_{i}, I_{0}, I_{1}, \ldots, I_{i}\right)-I\left(t ; t_{0}, t_{1}, \ldots t_{i-1}, I_{0}, I_{1}, \ldots, I_{i-1}\right) \\
& =I_{i}^{+} \exp \left(\int_{0}^{t-t_{i}} \alpha_{i}(\tau) d \tau\right)-I_{i-1}^{+} \exp \left(\int_{0}^{t-t_{i-1}} \alpha_{i-1}(\tau) d \tau\right) .
\end{aligned}
$$

Therefore,

$$
\begin{aligned}
\lim _{t \rightarrow \infty}\left(I\left(t ; t_{0}, t_{1}, \ldots, t_{i}, I_{0}, I_{1}, \ldots, I_{i}\right)-I\left(t ; t_{0}, t_{1}, \ldots t_{i-1}, I_{0}, I_{1}, \ldots, I_{i-1}\right)\right) \\
\quad=I_{i}^{+} \exp \left(\lim _{t \rightarrow \infty} \int_{0}^{t-t_{i}} \alpha_{i}(\tau) d \tau\right)-I_{i-1}^{+} \exp \left(\lim _{t \rightarrow \infty} \int_{0}^{t-t_{i-1}} \alpha_{i-1}(\tau) d \tau\right) \\
=I_{i}^{+} \exp \left(A_{i}\right)-I_{i-1}^{+} \exp \left(A_{i-1}\right) \\
>I_{\min } \exp \left(A_{i}\right)-I_{\max } \exp \left(A_{i-1}\right) \\
=I_{\min } \exp \left(A_{i-1}\right)\left(\exp \left(A_{i}-A_{i-1}\right)-\frac{I_{\max }}{I_{\min }}\right)>0 .
\end{aligned}
$$

The theorem is proved.

\section{CONCLUSIONS}

We will give an interpretation of the mathematical results obtained In the next few notes. 
Conclusion 1. Let us consider two variants of discreetly filling in the information.

Both variants do not differ in:

- The number filling in the external information (training);

- The storage coefficients of the information (the latter means that the variants refer to the same abstract individual).

Both variants are permissible to differ in:

- The initial value conditions - the initial moment and the initial information quantity in the human memory;

- The moments in which discrete fill-in of the information takes place;

- The size of changing the information in discrete trainings;

- The amounts of information after the last filling in the information.

Comparing both options after a sufficiently long period of time, we obtain that the option with a bigger amount of information since the last filling the amount of information has a bigger volume. (see Theorem 1). In other words, the amount of information in memory, calculated immediately after the last training is a key factor for the future development of information.

Conclusion 2. Look again two variants of discreetly filling the information.

Both variants do not differ in:

- The initial value conditions;

- The number filling of external information;

- The storage coefficients of information;

- The size of changing the information in discrete trainings;

- From the first to the penultimate moment, in which the discrete fillings of information are carried out.

Both variants are permissible to differ in:

- The last moment of training.

Then (after a sufficiently long period of time) the amount of information is larger in the case of the later filling in the quantity of the information (see Theorem 2).

Conclusion 3. Look again two variants of discreetly filling the information.

Both variants do not differ in:

- The initial value conditions;

- The storage coefficients of information;

- The size of changing the information in discrete trainings;

- From the first to the penultimate moment, in which the discrete fillings of information are carried out.

Both variants are permissible to differ in:

- The number of trainings.

Then (after a sufficiently long period of time) the amount of information is greater in the case of another (additional) filling in the quantity of information (see Theorem 3).

\section{Acknowledgements:}

1. The authors are grateful for the funding of this research to the Bulgarian National Scientific Research Fund, under contract DM19/1 (2017).

2. The authors are grateful for funding of this work under project BG05M2OP001-2.009-0015. 


\section{References}

1. Agarwal R., Hristova S., O'Regan D., Noninstantaneous impulses in Caputo fractional differential equations and practical stability via Lyapunov functions, Journal of the Franklin Institute, Vol. 354, Issue 7, (2017), 3097-3119.

2. Ahmad N., Ali Z., Shah K., Zada A., Ghaus ur Rahman, Analysis of implicit type nonlinear dynamical problem of impulsive fractional differential equations, Hindawi Complexity, Vol. 2018, Article ID 6423974, 15 pages.

3. Ali A., Rabiei F., Shah K., On Ulam's type stability for a class of impulsive fractional differential equations with nonlinear boundary conditions, J. of Nonlinear Sciences and Applications, Vol. 10, Issue 9, (2017), 4760-4775.

4. Alves J., Carvalho M., Siqueira J., Equilibrium states for impulsive semiflows, J. of Mathematical Analysis and Applications, Vol. 451, Issue 2, (2017), 839-857.

5. Chen $Z_{11}$ Zhang T., Tade M., Brooks G., Existence of periodic solutions of a continuous flow bioreactor model with impulsive control in microorganisms, J. of Applied Mathematics and Computing, Vol. 53, Issue 1-2, (2017), 471-486.

6. Chukleva R., Dishliev A., Dishlieva K., Continuous dependence of the solutions of the differential equations with variable structure and impulses in respect of switching functions International J. of Applied Science and Technology, Vol. 1, Issue 5, (2011), 46-59.

7. Dishliev A., Dishlieva K., Abou Habib M., Mathematical model of dynamics of the amount of information in human memory, American J. of Education, Vol. 123, Issue.4 (2), (2017), 1073-1081.

8. Dishliev A., Dishlieva K., Antonov A., Abou Habib M., Mathematical training model maintaining minimum amount of information in the memory, London Review of Education and Science, Vol. 22, Issue 2, (2017), 323-340.

9. Dishliev A., Bainov D., Continuous dependence on the initial condition of the solution of a system of differential equations with variable structure and with impulses, Putt. RIMS, Kyoto Univ., Vol. 23, (1987), 923-936.

10. Dishliev A., Dishlieva K., Antonov A., Abou Habib M., Mathematical model of the amount of information in the human memory for short time discrete trainings, Science and Educational Studies, Vol. VIII, Issue 1 (29), (2018), 229-244.

11. Dou J., Li S., Optimal impulsive harvesting policies for single-species populations, Applied Mathematics and Computation, Vol. 292, (2017), 145-155.

12. Longo F., IDE: Optimization problems and population dynamics, XI Congress GAFEVOL, University of Brasilia, Brazil, on October 23-26, (2017), 67-68.

13. Peng Y., Wu K., Qin S., Kang Y., Properties of solution of linear controlled systems with impulses at variable times, In Control Conference (CCC), 36th Chinese IEEE, (2017), 811-816.

14. Stamov G., Stamova I., On stable integral manifolds for impulsive Kolmogorov systems of fractional order, Modern Physics Letters B, Vol. 31, Issue 15 (2017), 1750168.

15. Tahmasebi A., Fodian A., Modification of homotopy perturbation method for solving system of integrodifferential equations, World J. of Modelling and Simulation, Vol. 13, Issue 2, (2017), 133-142.

16. Tripathy A., Santra S., Characterization of a class of second order neutral impulsive systems via pulsatile constant, Differential Equations \& Applications, Vol. 9, Issue 1, (2017), 87-98.

17. Witayakiattilerd W., PID controller singularly perturbing impulsive differential equations and optimal control problem, Advances in Mathematical Physics, Vol. 2017, Article ID 1938513.

18. Yang D., Wang JinRong, O'Regan D., Asymptotic properties of the solutions of nonlinear noninstantaneous impulsive differential equations, J. of the Franklin Institute Vol. 354, (2017), 6978-7011. 